\title{
NULL-CONTROLLABILITY OF ONE-DIMENSIONAL PARABOLIC EQUATIONS *
}

\author{
Giovanni Alessandrini $^{1}$ And Luis EscauriazA ${ }^{2}$
}

\begin{abstract}
We prove the interior null-controllability of one-dimensional parabolic equations with time independent measurable coefficients.
\end{abstract}

Mathematics Subject Classification. 35B37

Received June 12, 2006.

Published online October 13, 2007.

\section{INTRODUCTION}

Let us consider the following one-dimensional heat equation with variable coefficients

$$
\begin{cases}\partial_{x}\left(a(x) \partial_{x} z\right)+b(x) \partial_{x} z+c(x) z-\rho(x) \partial_{t} z=f \chi_{\omega}, & 0<x<1,0<t<T \\ z(0, t)=z(1, t)=0, & 0 \leq t \leq T \\ z(x, 0)=z_{0}, & 0 \leq x \leq 1\end{cases}
$$

Here, $z(x, t)$ is the state and $f(x, t)$ is an interior control that acts on the system over the open set $\omega \subset(0,1)$. The coefficients $a, b, c$ and $\rho$ are assumed to be measurable, bounded and for some $K \geq 1$,

$$
K^{-1} \leq \rho(x) \leq K, \quad K^{-1} \leq a(x) \leq K,|b(x)|+|c(x)| \leq K, \text { a.e. in }[0,1] .
$$

For any given $z_{0}$ in $L^{2}(0,1)$ and $f$ in $L^{2}(\omega \times[0, T])$, there is only one solution $z$ to $(1.1)$ in $C\left([0, T] ; L^{2}(0,1)\right) \cap$ $L^{2}\left(0, T ; H_{0}^{1}(0,1)\right)$. The goal of this paper is to analyze the interior null-controllability of (1.1). Specifically, we want to solve the following problem:

Given $T>0$ and $z_{0}$ in $L^{2}(0,1)$, to find $f$ in $L^{2}(\omega \times[0, T])$ such that the solution $z$ to (1.1) satisfies, $z(\cdot, T) \equiv 0$ in $(0,1)$.

In [11], it is shown that the system (1.1) is null-controllable at any positive time, when the coefficients $a$ and $\rho$ are Lipschitz in $[0,1]$. In this reference, the proof of null-controllability is based on an appropriate

\footnotetext{
Keywords and phrases. Null-controllability.

* The first author is supported in part by MIUR, PRIN n. 2004011204. The second author is supported by MEC grant, MTM2004-03029.

1 Dipartimento di Matematica e Informatica Università degli Studi di Trieste Via Valerio, 12/b 34127 Trieste, Italy; alessang@univ.trieste.it

2 Universidad del País Vasco / Euskal Herriko Unibertsitatea Dpto. de Matemáticas Apto. 644, 48080 Bilbao, Spain;

luis.escauriaza@ehu.es
} 
observability inequality for the adjoint system and it is implied by a global Carleman estimate. When the coefficients are smooth, the observability inequality can be proved introducing Fourier series and using high frequency asymptotic formulae for the eigenvalues of the corresponding Stürm-Liouville problem and classical results on the sums of real exponentials, see [16]. In [8] adopting the approach introduced by D.L. Russel in [18] (the null controllability of the wave operator at large times implies the null controllability of the heat equation at all times) it is shown that the system (1.1) is null controllable, when $a$ and $\rho$ have bounded variation in $[0,1]$.

The main result in this paper is the following.

Theorem 1. Assume that the coefficients $a, b, c$ and $\rho$ are bounded measurable and satisfy (1.2). Then, (1.1) is null-controllable at time $T$, for all $T>0$ and with controls $f$ in $L^{2}\left(0, T ; H_{0}^{1}(\omega)\right)$.

Let us briefly digress on some relevant consequences of this theorem. The interior null-controllability of (1.1) is known to imply the boundary null-controllability:

Given $T>0$ and $z_{0}$ in $L^{2}(0,1)$ there is $\alpha$ in $C_{0}^{\infty}((0, T])$ such that, the solution to

$$
\begin{cases}\partial_{x}\left(a(x) \partial_{x} z\right)+b(x) \partial_{x} z+c(x) z-\rho(x) \partial_{t} z=0, & 0<x<1,0<t<T \\ z(0, t)=\alpha(t), z(1, t)=0, & 0 \leq t \leq T \\ z(x, 0)=z_{0}, & 0 \leq x \leq 1\end{cases}
$$

verifies $z(T) \equiv 0$.

In fact, first we can let the system evolve freely until time $T / 2$ and replace $[0,1] \times(0, T)$ by $\left[-\frac{1}{2}, 1\right] \times(T / 2, T)$ in (1.1). Next, by Theorem 1, we can find an interior control of the new system over the region $\left(-\frac{1}{4},-\frac{1}{8}\right) \times$ $(T / 2, T)$ and take as boundary control the restriction of the controlled solution to $\{0\} \times[0, T]$. We can also infer results of observability. In fact, the $C^{\infty}$-interior regularity in the time-variable of solutions of parabolic equations with time-independent coefficients shows that the boundary control is in $C_{0}^{\infty}((0, T])$. The latter and [13] show that the operators mapping the initial data into the interior and boundary control are bounded from $L^{2}[0,1]$ into $L^{2}(\omega \times[0, T])$ and into $L^{\infty}(0, T)$ respectively. As is well known, these boundedness results, combined with standard duality arguments, imply two observability inequalities for the adjoint system:

There is $C=C(T, K)$ such that the inequalities

$$
\|\varphi(\cdot, 0)\|_{L^{2}(0,1)} \leq C\|\varphi\|_{L^{2}(\omega \times(0, T))},\|\varphi(\cdot, 0)\|_{L^{2}(0,1)} \leq C\left\|\partial_{x} \varphi(0, \cdot)\right\|_{L^{1}(0, T)}
$$

hold, when $\varphi$ is a solution of the adjoint system

$$
\begin{cases}\partial_{x}\left(a(x) \partial_{x} \varphi\right)-\partial_{x}(b(x) \varphi)+c(x) \varphi+\rho(x) \partial_{t} \varphi=0, & 0<x<1,0 \leq t<T \\ \varphi(0, t)=\varphi(1, t)=0, & 0 \leq t \leq T\end{cases}
$$

It also implies the uniform null-controllability of $1-d$ parabolic equations with rapidly oscillating periodic coefficients in a bounded interval (homogenization). Thus, extending previous results in [14], which needed more regular coefficients.

Returning to Theorem 1, in order to prove it, we proceed as follows. First, a change of variables shows that the internal controllability of the system (1.1) is equivalent to the same question for a system

$$
\begin{cases}\partial_{x}^{2} z-\rho(x) \partial_{t} z=f \chi_{\omega}, & 0<x<1,0<t<T \\ z(0, t)=z(1, t)=0, & 0 \leq t \leq T \\ z(x, 0)=z_{0}, & 0 \leq x \leq 1\end{cases}
$$

where $\rho$ is a new measurable function satisfying (1.2), for some new constant only depending on $K$, which we shall continue to denote by $K$. 
Then, if we denote by $\delta$ the inradius of the open set $\omega$, that is

$$
\delta=\sup \{r>0 \mid \exists \xi \in \omega,(\xi-r, \xi+r) \subset \omega\},
$$

and if $e_{1}, e_{2}, \ldots, e_{n}, \ldots$ and $0<\lambda_{1}^{2}<\lambda_{2}^{2}<\ldots<\lambda_{m}^{2} \ldots$ are respectively the eigenfunctions and eigenvalues of the Stürm-Liouville problem

$$
\left\{\begin{array}{l}
e^{\prime \prime}+\rho(x) \lambda^{2} e=0,0<x<1 \\
e(0)=e(1)=0
\end{array}\right.
$$

we prove the following:

Theorem 2. Assume that the coefficient $\rho$ satisfies (1.2). Then, there is a constant $N$, which depends on $K$ and on $\delta$ such that the inequality

$$
\sum_{\lambda_{k} \leq \mu} a_{k}^{2} \leq N \mathrm{e}^{N \mu} \int_{\omega}\left|\sum_{\lambda_{k} \leq \mu} a_{k} e_{k}\right|^{2} \mathrm{~d} x,
$$

holds for all $\mu \geq 1$ and all sequences $\left\{a_{k}\right\}$.

The analog of this inequality for the eigenfunctions of the Laplace-Beltrami operator on a compact and smooth Riemannian manifold with a possibly nonempty boundary was proved in [13]. There, G. Lebeau and L. Robbiano showed that it implies the interior null-controllability of the heat equation over the manifold by giving an explicit construction of the control function $f$ (see [14], Sect. 5, for a more simplified presentation).

The arguments in [13] show that the same iterative method of construction of the control function $f$ given in [13] works for the system (1.3), when Theorem 2 holds. Thus, Theorem 1 follows from Theorem 2.

For any given $z_{0}$ in $L^{2}(0,1)$ and $f$ in $L^{2}(\omega \times[0, T])$, there is only one solution $z$ to $(1.1)$ in $C\left([0, T] ; L^{2}(0,1)\right) \cap$ $L^{2}\left(0, T ; H_{0}^{1}(0,1)\right)$. The goal of this paper is to analyze the interior null-controllability of (1.1). Specifically, we want to solve the following problem:

Given $T>0$ and $z_{0}$ in $L^{2}(0,1)$, to find $f$ in $L^{2}(\omega \times[0, T])$ such that the corresponding solution $z$ to (1.1) satisfies, $z(\cdot, T) \equiv 0$ in $(0,1)$.

To prove Theorem 2 we start by following the arguments in [13]. In particular, given $\mu \geq 1$ and a sequence of real numbers $a_{1}, a_{2}, \ldots, a_{n}, \ldots$, we set

$$
u(x, y)=\sum_{\lambda_{k} \leq \mu} a_{k} e_{k}(x) \cosh \left(\lambda_{k} y\right) .
$$

This function satisfies

$$
\begin{cases}\partial_{x}^{2} u+\partial_{y}\left(\rho(x) \partial_{y} u\right)=0, & 0<x<1, y \in \mathbb{R} \\ u(0, y)=u(1, y)=0, & y \in \mathbb{R}, \\ \partial_{y} u(x, 0)=0, & 0<x<1,\end{cases}
$$

and the proof of Theorem 2 is a consequence of a quantification of the following qualitative result of unique continuation from the boundary:

Assume that $u$ satisfies $(1.7)$ and $u(x, 0) \equiv 0$, when $x$ is in $\omega \subset(0,1)$. Then, $u \equiv 0$ in $[0,1] \times \mathbb{R}$.

In [13], the one dimensional interval $[0,1]$ is replaced by a compact and smooth manifold $M, \partial_{x}^{2}$ by the corresponding Laplace-Beltrami operator on $M$ and the authors work out the quantification of a similar qualitative property of boundary unique continuation for the elliptic operator, $\triangle+\partial_{y}^{2}$, where $\triangle$ is the Laplace-Beltrami operator on $M$. To carry out this quantification they use two Carleman inequalities. Those methods require that the elliptic operator involved has Lipschitz second order coefficients and so, they can not be applied to the elliptic operator in (1.7), which has measurable coefficients. 
On the other hand, if $\sigma$ is a $2 \times 2$ symmetric and measurable matrix in the plane verifying the ellipticity condition

the weak solutions of the equation

$$
K^{-1}|\xi|^{2} \leq \sigma(x, y) \xi \cdot \xi \leq K|\xi|^{2}, \text { when }(x, y) \text { and } \xi \in \mathbb{R}^{2}
$$

$$
\nabla \cdot(\sigma(x, y) \nabla u)=0
$$

satisfy the strong unique continuation property:

If a $W_{\text {loc-solution of }}^{1,2}(1.9)$ on a connected open set $\Omega$ has a zero of infinite order at an interior point, then it must be zero.

See [2]. This qualitative result of strong unique continuation for uniformly elliptic equations in two independent variables is based on the connection between the solutions of these equations and the theory of quasiregular mappings [4] and on the so-called Ahlfors-Bers representation [1] of such mappings. Here, we describe some quantifications of this qualitative result and apply them to prove the null-controllability property. In particular, a "Hadamard's three circle theorem", Proposition 1, and a "doubling" type property, Proposition 2, adapted to the solutions of (1.9).

In Section 2 we recall the results we need from the theory of quasiregular mappings and prove the adapted Hadamard's three circle theorem and doubling property. In Section 3 we show how to apply them to prove Theorem 2, also using an estimate of continuation from Cauchy data for solutions of (1.9) Lemma 1, which we adapt from [3]. It may be worth noting that the approach used for the proof of Lemma 1, is based on a variation on the classical principle of majorization by harmonic measure [19], Chapter VIII, Section 1, p. 301, which in turn has its roots in arguments due to Carleman [6], pp. 3-4.

\section{Quantitative estimates of Unique CONTINUATION WITH DISCONTINUOUS COEFFICIENTS}

Throughout the paper, $z=x+i y, \Omega$ is a simply connected open set in the plane, $B_{r}$ a circle of radius $r$ centered at the origin, and

$$
\partial_{\bar{z}} f=\frac{1}{2}\left(\partial_{x} f+i \partial_{y} f\right), \partial_{z} f=\frac{1}{2}\left(\partial_{x} f-i \partial_{y} f\right)
$$

We shall denote by $C$ constants only depending on $K$, whereas by $N$ we shall denote constants only depending on $K$ and $\delta$.

When $u \in W_{l o c}^{1,2}(\Omega)$ is a weak solution to (1.9), and $\sigma$ satisfies (1.8) we can associate in a natural fashion, which generalizes the harmonic conjugate, a new function, the so called stream function $v$, which satisfies

$$
\nabla v=J \sigma \nabla u
$$

almost everywhere in $\Omega$ and is a weak solution to

$$
\nabla \cdot\left(\frac{\sigma}{\operatorname{det} \sigma} \nabla v\right)=0, \text { in } \Omega .
$$

Here $J$ denotes the matrix representing a $90^{\circ}$ rotation in the plane

$$
J=\left(\begin{array}{cc}
0 & -1 \\
1 & 0
\end{array}\right)
$$

Moreover, letting $f=u+i v$, we have $f \in W_{l o c}^{1,2}$ and satisfies

$$
\partial_{z} f=\mu \partial_{z} f+\nu \overline{\partial_{z} f}, \text { almost everywhere in } \Omega,
$$

where the complex valued functions $\mu$ and $\nu$ can be explicitly expressed in terms of $\sigma$, see [2], and verify

$$
|\mu|+|\nu| \leq \frac{K-1}{K+1}<1, \text { almost everywhere in } \Omega
$$


That is, $f$ is a $K$-quasiregular mapping.

To give an idea of why these results hold, observe that the vector field

$$
J \sigma \nabla u
$$

is, in the weak sense, curl-free in $\Omega$. To verify that $v$ is a $W_{l o c}^{1,2}$-solution of $(2.2)$, observe that, from (2.1), one obtains that the vector field

is, in the weak sense, divergence-free in $\Omega$.

$$
\frac{\sigma}{\operatorname{det} \sigma} \nabla v=J \sigma^{-1} J^{t} \nabla v
$$

By the Ahlfors-Bers representation [1] (see also [4] and [5], Chap. II.6, pp. 258-259), any K-quasiregular mapping $f$ in $B_{1}$ can be written as

$$
f=F \circ \chi
$$

where $F$ is holomorphic in $B_{1}$ and $\zeta=\chi(z)$ is a $K$-quasiconformal homeomorphism from $B_{1}$ onto $B_{1}$, which verifies, $\chi(0)=0, \chi(1)=1$,

$$
C^{-1}\left|z_{1}-z_{2}\right|^{\frac{1}{\alpha}} \leq\left|\chi\left(z_{1}\right)-\chi\left(z_{2}\right)\right| \leq C\left|z_{1}-z_{2}\right|^{\alpha}, \text { when } z_{1}, z_{2} \in B_{1}
$$

for some $0<\alpha<1$ and $C \geq 1$ depending only on $K$.

We now recall the Hadamard's three-circle theorem [17].

Theorem 3. Let $F$ be a holomorphic function of a complex variable in the ball $B_{r_{2}}$ and $M(r)=\max _{B_{r}}|F|$. Then, the following is valid for $0<r_{1} \leq r \leq r_{2}$,

$$
\log M(r) \leq \frac{\log \frac{r_{2}}{r}}{\log \frac{r_{2}}{r_{1}}} \log M\left(r_{1}\right)+\frac{\log \frac{r}{r_{1}}}{\log \frac{r_{2}}{r_{1}}} \log M\left(r_{2}\right) .
$$

The meaning of this inequality is that $\log M(r)$ is a convex function of the variable $\log r$.

Let $u \in W_{\text {loc }}^{1,2}\left(B_{R}\right)$ be a weak solution to $(1.9)$ and let $f: B_{R} \longrightarrow \mathbb{C}$ be the associated $K$-quasiregular mapping. Rescaling (2.5) we have that $f=F \circ \chi$, where $F$ is holomorphic in $B_{R}$ and $\chi: B_{R} \longrightarrow B_{R}$ is a $K$-quasiconformal homeomorphism, which verifies

$$
R C^{-1}\left|\frac{z}{R}\right|^{\frac{1}{\alpha}} \leq|\chi(z)| \leq R C\left|\frac{z}{R}\right|^{\alpha}
$$

where $C$ is the same as in (2.5).

Define

and

$$
\mathcal{B}_{r}=\left\{z \in B_{R}:|\chi(z)|<r\right\}
$$

$$
m(r)=\max _{\mathcal{B}_{r}}|f(z)|, \text { when } r<R .
$$

Then, through the change of coordinates, $\zeta=\chi(z)$, the Hadamard's three circle theorem takes the form: the function $\log m(r)$ is a convex function of $\log r$,

$$
\log m(r) \leq \frac{\log \frac{r_{2}}{r}}{\log \frac{r_{2}}{r_{1}}} \log m\left(r_{1}\right)+\frac{\log \frac{r}{r_{1}}}{\log \frac{r_{2}}{r_{1}}} \log m\left(r_{2}\right), \text { when } 0<r_{1} \leq r \leq r_{2}<R,
$$

and the sets $\mathcal{B}_{r}$, almost look like balls. In particular,

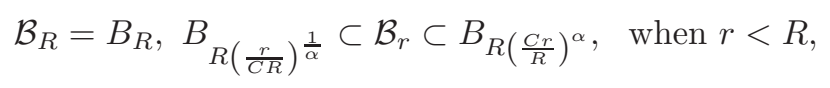

and $C$ is the same constant appearing in (2.6). Note incidentally that (2.9) implies a weak unique continuation property, that is, if $m\left(r_{1}\right)=0$ for some small $r_{1}$, then $m(r)=0$ for all $r<R$. 
On the other hand, the difference quotients of convex functions are nondecreasing functions of their arguments. This implies that, if $f$ is not identically zero,

$$
\frac{\log m\left(\frac{r}{2}\right)-\log m\left(\frac{r}{4}\right)}{\log \frac{r}{2}-\log \frac{r}{4}} \leq \frac{\log m\left(\frac{R}{2}\right)-\log m\left(\frac{R}{4}\right)}{\log \frac{R}{2}-\log \frac{R}{4}}, \quad \text { when } r \leq R,
$$

and thus,

$$
\frac{m\left(\frac{r}{2}\right)}{m\left(\frac{r}{4}\right)} \leq \frac{m\left(\frac{R}{2}\right)}{m\left(\frac{R}{4}\right)}, \text { when } r<R .
$$

We may prescribe that the the stream function $v$ of $u$ satisfies $v(0)=0$. We have that $F=u+i v$ is holomorphic in the $\zeta=\xi+i \eta$ coordinates in $B_{R}$, hence, solving the Cauchy-Riemann equations,

$$
v(\xi, \eta)=\int_{0}^{\eta} u_{\xi}(\xi, s) \mathrm{d} s-\int_{0}^{\xi} u_{\eta}(t, 0) \mathrm{d} t, \text { in } B_{R} .
$$

This formula and interior estimates for harmonic functions [10] show that in the $\zeta$-coordinates we have,

$$
\|u\|_{L^{\infty}\left(B_{r}\right)} \leq \max _{B_{r}}|F(\zeta)| \leq C\|u\|_{L^{\infty}\left(B_{2 r}\right)}, \text { when } r \leq \frac{R}{2},
$$

where $C>0$ is an absolute constant. In the $z$-coordinates, the last inequality reads as

$$
\|u\|_{L^{\infty}\left(\mathcal{B}_{r}\right)} \leq \max _{\mathcal{B}_{r}}|f(z)| \leq C\|u\|_{L^{\infty}\left(\mathcal{B}_{2 r}\right)}, \text { when } r \leq \frac{R}{2},
$$

and from (2.12), (2.9) we obtain:

Proposition 1. Let $u \in W_{\text {loc }}^{1,2}\left(B_{R}\right)$ be a weak solution to (1.9) and let $\mathcal{B}_{r}, 0<r \leq R$ be the open sets introduced in (2.7), then we have

$$
\|u\|_{L^{\infty}\left(\mathcal{B}_{\frac{r}{2}}\right)} \leq C\|u\|_{L^{\infty}\left(\mathcal{B}_{r_{1}}\right)}^{\theta}\|u\|_{L^{\infty}\left(\mathcal{B}_{r_{2}}\right)}^{1-\theta}, \text { when } r_{1} \leq r \leq r_{2}<R, \theta=\frac{\log \frac{r_{2}}{r}}{\log \frac{r_{2}}{r_{1}}}
$$

And also, from (2.11):

Proposition 2. Let $u$ and $\mathcal{B}_{r}$ be as above. If $u$ is not identically zero, then we have

$$
\frac{\|u\|_{L^{\infty}\left(\mathcal{B}_{r}\right)}}{\|u\|_{L^{\infty}\left(\mathcal{B}_{\frac{r}{2}}\right)}} \leq C \frac{\|u\|_{L^{\infty}\left(\mathcal{B}_{R}\right)}}{\|u\|_{L^{\infty}\left(\mathcal{B}_{\frac{R}{4}}\right)}} \text {, when } r \leq R \text {. }
$$

These are respectively a Hadamard's three circle theorem and a doubling property adapted to the solution $u$ through the family of "balls" $\mathcal{B}_{r}$.

\section{Proof of Theorem 2}

First let us note that replacing $z$ in (1.1) by $z_{1}=\mathrm{e}^{-K^{2} t} z$, we have

$$
\partial_{x}\left(a(x) \partial_{x} z_{1}\right)+b(x) \partial_{x} z_{1}+\left(c(x)-K^{2} \rho(x)\right) z_{1}-\rho(x) \partial_{t} z_{1}=\mathrm{e}^{-K^{2} t} f \chi_{\omega} .
$$

Introducing

$$
B(x)=\int_{0}^{x} \frac{b(s)}{a(s)} \mathrm{d} s
$$


we observe that (3.1) can be rewritten as

$$
\mathrm{e}^{-B(x)} \partial_{x}\left(a(x) \mathrm{e}^{B(x)} \partial_{x} z_{1}\right)+\left(c(x)-K^{2} \rho(x)\right) z_{1}-\rho(x) \partial_{t} z_{1}=\mathrm{e}^{-K^{2} t} f \chi_{\omega} .
$$

Because $c-K^{2} \rho$ is nonpositive in $[0,1]$, the solution $w$ to

$$
\left\{\begin{array}{l}
\mathrm{e}^{-B(x)} \frac{\mathrm{d}}{\mathrm{d} x}\left(a(x) \mathrm{e}^{B(x)} \frac{\mathrm{d} w}{\mathrm{~d} x}\right)+\left(c(x)-K^{2} \rho(x)\right) w=0 \\
w(0)=w(1)=1
\end{array}\right.
$$

verifies $0<w(x) \leq 1$ in $[0,1]$ and replacing $z_{1}$ with the new dependent variable $z_{2}=z_{1} / w$, we have

$$
\mathrm{e}^{-B(x)} \partial_{x}\left(a(x) w^{2}(x) \mathrm{e}^{B(x)} \partial_{x} z_{2}\right)-\rho(x) w^{2}(x) \partial_{t} z_{2}=w(x) f \mathrm{e}^{-K^{2} t} \chi_{\omega} .
$$

Setting

$$
\begin{aligned}
& L=\int_{0}^{1} a^{-1}(s) w^{-2}(s) \mathrm{e}^{-B(s)} \mathrm{d} s, y=\frac{1}{L} \int_{0}^{x} a^{-1}(s) w^{-2}(s) \mathrm{e}^{-B(s)} \mathrm{d} s, \\
& \tilde{\rho}(y)=L^{2} a(x) w^{4}(x) \mathrm{e}^{2 B(x)} \rho(x), \tilde{f}(y, t)=L^{2} a(x) w^{3}(x) \mathrm{e}^{2 B(x)} f(x, t),
\end{aligned}
$$

and writing

$$
\tilde{z}(y, t)=z_{2}(x, t), \chi_{\tilde{\omega}}(y)=\chi_{\omega}(x),
$$

the new function $\tilde{z}$, is a solution of the system

$$
\begin{cases}\partial_{y}^{2} \tilde{z}-\tilde{\rho}(y) \partial_{t} \tilde{z}=\tilde{f}(y, t) \chi_{\tilde{\omega}}(y), & 0<y<1,0<t<T \\ \tilde{z}(0, t)=\tilde{z}(1, t)=0, & 0 \leq t \leq T \\ \tilde{z}(y, 0)=z_{0}(x) / w(x), & 0 \leq y \leq 1\end{cases}
$$

All together,

$$
\tilde{z}(y, t)=\mathrm{e}^{-K^{2} t} z(x, t) / w(x)
$$

and if we rename the new variables, sets or functions $\tilde{z}, y, \tilde{\omega}, \tilde{\rho}, \tilde{f}$ and $z_{0} / w$ as $z, x, \omega, \rho, f$ and $z_{0}$ respectively, the new function $z$ is a solution to a system of the form (1.3).

Considering the associated Stürm-Liouville problem (1.5), we extend the eigenfunctions $e_{j}, j \geq 1$, to $[-1,1]$ by an odd reflection in 0 , similarly we extend $\rho$ by an even reflection in 0 . Next, we continue these new functions to all of $\mathbb{R}$ as periodic functions of period 2. The extended $\rho$ verifies $(1.2), e_{j} \in C^{1,1}(\mathbb{R})$ and $e_{j}^{\prime \prime}+\rho(x) \lambda_{j}^{2} e_{j}=0$, almost everywhere in $\mathbb{R}$.

Being the change of variable $y=y(x)$ bi-Lipschitz, with Lipschitz constants which only depend on $K$, the open set $\omega$ is transformed into a new open subset of $(0,1)$ whose inradius is comparable to $\delta$. We continue to denote the transformed set and its inradius by $\omega$ and $\delta$, respectively. Also we can assume, up to a translation along the real line, $(-\delta, \delta) \subset \omega \subset(-1,1)$.

Given $\mu \geq 1$ and a sequence of real numbers $a_{1}, a_{2}, \ldots a_{n}, \ldots$, the function

$$
u(x, y)=\sum_{\lambda_{k} \leq \mu} a_{k} e_{k}(x) \cosh \left(\lambda_{k} y\right),
$$

verifies

$$
\left\{\begin{array}{l}
\partial_{x}^{2} u+\partial_{y}\left(\rho(x) \partial_{y} u\right)=0, \text { in } \mathbb{R}^{2} \\
\partial_{y} u(x, 0)=0, \text { in } \mathbb{R}
\end{array}\right.
$$


and its stream function $v$ can be chosen so that, $v \in W_{l o c}^{1,2}\left(\mathbb{R}^{2}\right)$,

$$
\left\{\begin{array} { l } 
{ \partial _ { x } v = - \rho ( x ) \partial _ { y } u , } \\
{ \partial _ { y } v = \partial _ { x } u , }
\end{array} \quad \text { and } \quad \left\{\begin{array}{l}
\partial_{x}\left(\frac{1}{\rho(x)} \partial_{x} v\right)+\partial_{y}^{2} v=0, \text { in } \mathbb{R}^{2} \\
v(x, 0)=0, \text { in } \mathbb{R} .
\end{array}\right.\right.
$$

Let $f=u+i v$, consider the family of "balls" $\mathcal{B}_{r}$ associated to $f$ in Section 2 at scale $R$ and choose $R=2(4 C)^{\frac{1}{\alpha}}$, where $\alpha$ and $C$ are the constants in (2.6) and (2.10). With this choice, $\mathcal{B}_{\frac{R}{4}} \supset B_{2}$. The interior bounds for subsolutions of elliptic equations [10] give

$$
\|u\|_{L^{\infty}\left(B_{R}\right)} \leq \frac{C}{R}\|u\|_{L^{2}\left(B_{2 R}\right)} .
$$

These and the orthogonality of the eigenfunctions $e_{j}, j \geq 1$, imply that

$$
\frac{\|u\|_{L^{\infty}\left(\mathcal{B}_{R}\right)}}{\|u\|_{L^{\infty}\left(\mathcal{B}_{\frac{R}{4}}\right)}} \leq \mathrm{e}^{C \mu}
$$

An iteration of (2.14) and (3.3) give

$$
\|u\|_{L^{\infty}\left(\mathcal{B}_{R}\right)} \leq \mathrm{e}^{C k \mu}\|u\|_{L^{\infty}\left(\mathcal{B}_{\frac{R}{2^{k}}}\right)}, \text { when } k \geq 1
$$

and from (2.10), there is $k=k(\delta, K)$ such that, $\mathcal{B}_{\frac{R}{2^{k}}} \subset B_{\frac{\delta}{2}}$. Thus,

$$
\|u\|_{L^{\infty}\left(B_{1}\right)} \leq \mathrm{e}^{N \mu}\|u\|_{L^{\infty}\left(B_{\frac{\delta}{2}}\right)}
$$

The following inequality, which is an estimate on the continuation from Cauchy data, holds.

Lemma 1. There are constants $0<\theta<1$ and $C>0$, only depending on $K$, such that the inequality

$$
\|u\|_{L^{\infty}\left(B_{\frac{r}{2}}\right)} \leq C r^{-\frac{\theta}{2}}\|u(\cdot, 0)\|_{L^{2}(-r, r)}^{\theta}\|u\|_{L^{\infty}\left(B_{4 r}\right)}^{1-\theta}
$$

holds, when $r \leq 1$.

The Lemma and (3.4) give

$$
\|u\|_{L^{\infty}\left(B_{1}\right)} \leq N \mathrm{e}^{N \mu}\|u(\cdot, 0)\|_{L^{2}(-\delta, \delta)},
$$

which proves Theorem 2 .

Proof of Lemma 1. The proof is essentially contained in [3], Theorem 4.5. For the sake of completeness we summarize here the argument.

Recalling that $f=u+i v$ is analytic in the $\zeta$-variable, we have that $\log |f(\zeta)|$ is subharmonic in $B_{R}$ and consequently one can verify that $\log |f(z)|$ is a subsolution for an elliptic operator in divergence form $E$ with a matrix of coefficients verifying (1.8). For $r>0$, let $w$ be the solution to

$$
\left\{\begin{array}{l}
E w=0, \quad \text { in } B_{r}^{+} \\
w=1, \quad \text { in }(-r, r) \\
w=0, \quad \text { in } \partial B_{r}^{+} \backslash(-r, r) .
\end{array}\right.
$$


On $\partial B_{r}^{+}$we have

$$
\log |f| \leq w \log \|u(\cdot, 0)\|_{L^{\infty}(-r, r)}+(1-w) \log \|f\|_{L^{\infty}\left(B_{r}\right)}
$$

and the maximum principle implies that the same inequality also holds in $B_{r}^{+}$. The Hölder continuity at the boundary of $w$ and the Harnack's inequality [10] show that there is $\eta \in(0,1)$, which only depends on $K$, such that $w(z) \geq \eta$ in $B_{\frac{r}{2}}^{+}$.

Using $v(0)=0,(2.1)$ and interior bounds for elliptic equations [10], we have

$$
\|v\|_{L^{\infty}\left(B_{r}\right)} \leq C\|\nabla v\|_{L^{2}\left(B_{2 r}\right)} \leq C\|\nabla u\|_{L^{2}\left(B_{2 r}\right)} \leq C\|u\|_{L^{\infty}\left(B_{4 r}\right)} .
$$

These imply

$$
\|u\|_{L^{\infty}\left(B_{\frac{r}{2}}\right)} \leq C\|u(\cdot, 0)\|_{L^{\infty}(-r, r)}^{\eta}\|u\|_{L^{\infty}\left(B_{4 r}\right)}^{1-\eta} .
$$

For every $\alpha \in(0,1]$, we have the interpolation inequality

$$
\|\varphi\|_{L^{\infty}(-r, r)} \leq C\left(\|\varphi\|_{L^{2}(-r . r)}^{\beta}|\varphi|_{C^{\alpha}(-r, r)}^{1-\beta}+r^{-\frac{1}{2}}\|\varphi\|_{L^{2}(-r . r)}\right),
$$

where $\beta=\frac{2 \alpha}{1+2 \alpha}, C>0$ only depends on $\alpha$ and $|\varphi|_{C^{\alpha}(-r, r)}$ denotes the standard $C^{\alpha}$ seminorm. Next, we use the interior Hölder bound for $u$, [10],

$$
|u|_{C^{\alpha}\left(B_{r}\right)} \leq C r^{-\alpha}\|u\|_{L^{\infty}\left(B_{4 r}\right)},
$$

with $C>0$ and $\alpha \in(0,1]$ only depending on $K$. Combining (3.6) with (3.7) and (3.8), we obtain the thesis with $\theta=\beta \eta$.

The interpolation inequality (3.7) can be proved essentially along the same lines as the interpolation inequalities in [10], Section 6.8.

\section{REFERENCES}

[1] L. Ahlfors and L. Bers, Riemann's mapping theorem for variable metrics. Ann. Math. 72 (1960) 265-296.

[2] G. Alessandrini and R. Magnanini, Elliptic equations in divergence form, geometric critical oints of solutions and Stekloff eigenfunctions. SIAM J. Math. Anal. 25 (1994) 1259-1268.

[3] G. Alessandrini and L. Rondi, Stable determination of a crack in a planar inhomogeneous conductor. SIAM J. Math. Anal. 30 (1998) 326-340.

[4] L. Bers and L. Nirenberg, On a representation theorem for linear elliptic systems with discontinuous coefficients and applications, in Convegno Internazionale sulle Equazioni alle Derivate Parziali, Cremonese, Roma (1955) 111-138.

[5] L. Bers, F. John and M. Schechter, Partial Differential Equations. Interscience, New York (1964).

[6] T. Carleman, Les Fonctions Quasi Analytiques. Gauthier-Villars, Paris (1926).

[7] C. Castro and E. Zuazua, Concentration and lack of observability of waves in highly heterogeneous media. Arch. Rat. Mech. Anal. 164 (2002) 39-72.

[8] E. Fernandez-Cara and E. Zuazua, On the null controllability of the one-dimensional heat equation with BV coefficients. Comput. Appl. Math. 21 (2002) 167-190.

[9] A.V. Fursikov and O. Yu. Imanuvilov, Controllability of evolution equations. Lecture Notes Series 34, Research Institute of Mathematics, Global Analysis Research Center, Seoul National University (1996).

[10] D. Gilbarg and N.S. Trudinger, Elliptic Partial Differential Equations of Second Order, 2nd edn., Springer-Verlag, BerlinHeildeberg-New York-Tokyo (1983).

[11] O.Yu. Imanuvilov and M. Yamamoto, Carleman estimate for a parabolic equation in Sobolev spaces of negative order and its applications, in Control of Nonlinear Distributed Parameter Systems, G. Chen et al. Eds., Marcel-Dekker (2000) 113-137.

[12] E.M. Landis and O.A. Oleinik, Generalized analyticity and some related properties of solutions of elliptic and parabolic equations. Russian Math. Surv. 29 (1974) 195-212.

[13] G. Lebeau and L. Robbiano, Contrôle exact de l'équation de la chaleur. Commun. Partial Differ. Equ. 20 (1995) $335-356$.

[14] G. Lebeau and E. Zuazua, Null controllability of a system of linear thermoelasticity. Arch. Rat. Mech. Anal. 141 (1998) 297-329. 
[15] F.H. Lin, A uniqueness theorem for parabolic equations. Comm. Pure Appl. Math. 42 (1988) 125-136.

[16] A. López and E. Zuazua, Uniform null-controllability for the one-dimensional heat equation with rapidly oscillating periodic density. Ann. I.H.P. - Analyse non linéaire 19 (2002) 543-580.

[17] A.I. Markushevich, Theory of Functions of a Complex Variable. Prentice Hall, Englewood Cliffs, NJ (1965).

[18] D.L. Russel, A unified boundary controllability theory for hyperbolic and parabolic partial differential equations. Stud. Appl. Math. 52 (1973) 189-221.

[19] M. Tsuji, Potential Theory in Modern Function Theory. Maruzen, Tokyo (1959).

[20] I.N. Vekua, Generalized Analytic Functions. Pergamon, Oxford (1962). 